\title{
EXPERIMENT AND MODEL-BASED INVESTIGATION OF COMMINUTION IN A HAMMER MILL
}

\author{
S. NAIK ${ }^{1}$, Y. FENG ${ }^{2} \&$ B. CHAUDHURI ${ }^{1,3}$ \\ ${ }^{1}$ Department of Pharmaceutical Sciences, University of Connecticut, USA. \\ ${ }^{2}$ Eli Lilly and Company, USA. \\ ${ }^{3}$ Institute of Material Sciences, University of Connecticut, USA.
}

\begin{abstract}
Particle size reduction of dry granular material by mechanical means, also known as milling or comminution, is undoubtedly a very important unit operation in pharmaceutical, agricultural, food, mineral and paper industries. As comminution is a stochastic and a nonlinear process, an attempt was made to understand this complicated process by conducting parametric studies experimentally and computationally using discrete element method (DEM). Greater size reduction was observed at higher rotational speed of the hammer owing to the greater centrifugal force experienced by the particles. Increase in impeller wall tolerance resulted in rolling mode regime of powder bed, which was found to be significant at low impeller speeds. A numerical model based on DEM was used to simulate a hammer mill and study the breakage and kinematics of the particle motion within the hammer mill. In the simulations, increase in hammer tip speed causes higher frequency of impact of particles per unit time and higher specific energy of impact resulting in generation of much finer end product. A limit can be conceived during the breakage event. This is because as size reduction occurs, the breakage rates can fall for very fine particles as crack propagation ceases. This is because as size decreases the probability of finding a flaw also decreases. Simulations also showed a higher milling rate for big hammers as larger hammers decrease the tolerance between the milling chamber and rotating impeller. To study the effect of material properties, the energy of fragmentation was estimated and it was found to increase as the material became more cohesive.
\end{abstract}

Keywords: DEM, fragmentation, mill, pharmaceutical.

\section{INTRODUCTION}

Normally, pharmaceutical powders are polydisperse, i.e. consisting of particles of different sizes. Polydisperse powders create considerable difficulties in the production of dosage forms. Particles of monosize (equal size) may be ideal for pharmaceutical purposes. In practice, powders with narrow range of size distribution can obviate the problems in processing them further. Size reduction alone is not sufficient to obtain mono-size or narrow-size range powder. Therefore, size reduction and size separation should be combined to obtain powders of desired size. There are numerous industries that depend on size reduction to improve the performance or to meet the specifications. The chemical, pharmaceutical, food and mining industries all rely on size reduction. Its uses include grinding polymers for recycling, improving extraction of a valuable constituent from ores, facilitating separation of grain components, boosting the biological availability of medications and producing particles of an appropriate size for a given use. There are many types of size-reduction equipment, which are often developed empirically to handle specific materials and then are applied in other situations. Knowing the properties of the material relevant to breakage is essential. Probably, the most important characteristic governing size reduction is hardness because almost all size-reduction techniques involve somehow creating new surface area, and this requires adding energy proportional to the bonds holding the feed particles together. Flow properties can be major factors too, because many size-reduction processes are continuous, 
but often have choke points at which bridging and flow interruption can occur. Size reduction process is also termed as comminution or diminution or pulverization. Normally, size reduction may be achieved by two methods, namely by precipitation and by mechanical process. In the precipitation method, the substance is dissolved in an appropriate solvent. This method is suitable for the production of raw materials and bulk drugs. Inorganic chemicals, such as calcium carbonate, magnesium carbonate and yellow mercuric oxide, are prepared by precipitation method. In the mechanical process, the substance is subjected to mechanical forces using grinding equipment. The most commonly used mills are the rotary cutter, hammer mill, roller mill, ball mills and fluid energy mills, used in various stages of manufacturing. Size reduction is generally achieved by particle fracturing and subsequent fragmentation under the action of externally applied energy. The surface characteristic and fracture pattern under an applied energy is not only influenced by the material properties but also by the operational conditions of the hammer mill. The knowledge of particle flow, granular fracturing and size reduction in a mill is thus very critical to optimize and to scale up this operation. Substantial effort has been expended in last three decades to understand the milling performance as a function of the material properties, operational conditions and machine configuration. Several experimental and mathematical approaches to understand the milling behavior in Ball Mill have been performed [1-6]. Unlike population-based models that ignore particle-particle and particle-wall fragmentation, DEM explicitly considers interparticle and particle-boundary interactions thereby providing an effective tool to model fragmentation. Experiments and discrete element method (DEM)-based modeling of milling of pharmaceutical excipients in a very small-scale oscillatory single ball mill was performed by Kwan et al. [7]. Campbell et al. [8] developed the relationship between the inlet and outlet particle size distribution in a roller milling operation [8]. The impact mills have been investigated by Austin [9,10], Vogel and Peukert [11] and Djordjevic et al. [12] for different materials. Austin developed simplified analytical model of high-speed milling of limestone, cement; whereas PMMA/glass and rock were the materials of interest for Vogel and Djordjevic. A comprehensive understanding of the fragmentation mechanism from experiments facilitated the development of a series of mechanistic and population balance based models [9-11]. Comprehensive modeling of milling based on first principles was attempted by Djordjevic et al. [12] using DEM. This DEM-based direct modeling of the milling process was limited to a small number of spherical particles. To the best of our knowledge, no previous work has exploited the 3D DEM to study the granular milling of pharmaceutical granules in a hammer mill. In this article, an experimental and 3D DEM modeling based method were used to gain insight into the behavior of pharmaceutical powders in the hammer mill. The effect of material properties and the operational conditions on the milling performance was also determined.

\section{EXPERIMENTAL METHOD}

A variable speed, digitally controlled, direct drive Wiley Mill, procured from Thomas Scientific (Swedesboro, NJ), was used in our experimental milling study. The mill provides continuous variation of rotational speeds (of hammers) from 600 to $1140 \mathrm{rpm}$ with constant torque maintained throughout the speed range. Parametric studies were conducted to study the effect of speed, impeller wall tolerance and feed rate on particle size reduction. The experiments were performed using lactose nonpareils. In our milling experiments, the material is fed at the top center, thrown out centrifugally at first impact with the hammers and ground by impact against the wall or cut due to the presence of hammers at the periphery. The 
Table 1: Conditions examined for the milling experiments with lactose.

\begin{tabular}{ll}
\hline Parameters & Values \\
\hline Speed & $600-1140 \mathrm{rpm}$ \\
Clearance & $2.9-3.7 \mathrm{~mm}$ \\
Feed rate & $60-200 \mathrm{~g} / \mathrm{min}$ \\
\hline
\end{tabular}

material is retained until it is small enough to fall through the screen that forms the lower portion of the casing. Particles fine enough to pass through the screen are discharged almost as fast as they are formed in a glass container. Sampling was performed at regular intervals for example, $5 \mathrm{~s}$ for the first four samples, $10 \mathrm{~s}$ for the next four samples and $20 \mathrm{~s}$ for the next samples. The experimental factors were studied at 2-3 levels. The average particle sizes for the entire sample are calculated from sieve analysis using Rotap Sieve Analyser. Particle shape analysis was performed by the Optical Microscope-Camera (Olympus SZ61) and Image Analysis software (Image Pro Plus). The effect of the operating conditions examined using the mill is listed in Table 1.

\section{COMPUTATIONAL METHOD}

The DEMs, originally developed by Cundall and Strack $[13,14]$, were used to simulate the dynamic behavior of granular materials inside the hammer mill. It has been successfully used to simulate chute flow, hopper discharge and flows in rotating drums [15-17]. In this model, the granular material is considered as a collection of frictional inelastic spherical particles. Each particle interacts with its neighbors or with the boundary only at contact points through normal and tangential forces. The forces and torques acting on each of the particles are calculated in the following way:

$$
\begin{gathered}
\sum F_{\mathrm{i}}=\mathrm{m}_{\mathrm{i}} \mathrm{g}+F_{\mathrm{N}}+F_{\mathrm{T}}+F_{\text {cohes }} \\
\sum T_{\mathrm{i}}=r_{\mathrm{i}} \times F_{\mathrm{T}}
\end{gathered}
$$

The force on each particle is given by the sum of gravitational, interparticle (normal and tangential $F_{\mathrm{N}}$ and $\left.F_{\mathrm{T}}\right)$ and cohesive forces $\left(F_{\text {cohes }}\right)$ as indicated in eqns (1) and (2). The corresponding torque on each particle is the sum of the moment of the tangential forces $\left(F_{\mathrm{T}}\right)$ arising from interparticle contacts (eqn (2)). Each particle may interact with its neighbors or with the boundary only at contact points through normal and tangential forces. The normal forces $\left(F_{\mathrm{N}}\right)$ and the tangential forces $\left(F_{\mathrm{T}}\right)$ in interparticle or particle-wall collision were calculated with the 'latching spring model' and 'incrementally slipping model', respectively, developed by Walton [18]. The normal forces between pairs of particles in contact are defined using a spring with constants $K_{1}$ and $K_{2}$ for compression and recovery: $F_{\mathrm{N}}=K_{1}$ (for compression) and $F_{\mathrm{N}}=K_{2}\left(\mu_{1}-\mu_{2}\right)$ (for recovery). Here $i$ represents the overlap during contact. The degree of inelasticity of collisions is incorporated in this model by including a coefficient of restitution $e=\left(K_{1} / K_{2}\right)^{1 / 2}(0<e<1$, where $e=1$ implies perfectly elastic collision with no energy dissipation, $e=0$ implies completely inelastic collision). Tangential forces $\left(F_{\mathrm{T}}\right)$ in interparticle or particle-wall collision are calculated employing Walton's incrementally slipping model. After contact occurs, tangential forces build up, causing displacement in the tangential plane of contact. The initial tangential stiffness is considered a fraction of the normal stiffness. In this model, the static frictional limit was considered to follow 
Coulomb-Amontons's law, i.e. if $F_{\mathrm{T}}<\mu_{s} F_{N}\left(i_{\mathrm{S}}\right.$ is the static friction coefficient and $F_{N}$ is the total normal force) [18], equal to the cohesive force plus the static weight of particles transmitted through the bed), no relative motion between particles occurred, while if the particles are in relative motion with respect to one another, $F_{\mathrm{t}}=\mu_{\mathrm{s}} F_{\mathrm{n}}$. This model takes into account the elastic deformations that can occur in the tangential directions of the contacts. The tangential force $T$ is evaluated considering an effective tangential stiffness $k_{\mathrm{T}}$ associated with a linear spring. It is incremented at each time step as $T_{\mathrm{t}+1}=T_{t}+k_{T} \Delta \mathrm{s}$, where $\Delta \mathrm{s}$ is the relative tangential displacement between two time steps. For details on the definition of $\Delta \mathrm{s}$, see Walton [18]. For simplicity, in this paper, the static and dynamic friction coefficients were considered equal value in the simulations.

Cohesion $\left(F_{\text {cohes }}\right)$ was modeled (eqn (3)) following the square-well type forces by Walton [19] and Chaudhuri et al. [20] in which the granular bond number $(K)$ and frictional parameters are varied to simulate the cohesive behavior of various pharmaceutical excipients (MCC, lactose). This constant force lumps all short range effects such as electrostatic or van der Waals forces

$$
F_{\text {cohesion }}=K \cdot \mathrm{mg}
$$

The cohesive force eqn (3) is applied between contacting particles or particle and wall. Different values of $K$ used for this study are listed in Table 2.

The major computational tasks of DEM in each time step are as follows: (i) add/delete contact between particles thus updating neighbor lists, (ii) compute contact forces from contact properties, (iii) sum all forces and torques on particles and update position and (iv) determine the trajectory of the particle by integrating Newton's laws of motion (second-order scalar equations in three dimensions) using a central difference scheme, Verlet's Leap Frog method. The DEM code was written in C language and was run in 32- and 64-bit Linux clusters.

To understand the breakage mechanism, the intensity of dynamic stress induced by the impeller due to impact was calculated using a simple equation

$$
S=\rho V_{p} V_{p p}
$$

where $S$ is the induced stress, $V_{\mathrm{p}}$ is the propagation velocity $(\mathrm{m} / \mathrm{s})$ of longitudinal elastic waves in the material, a material property, $V_{\mathrm{pp}}$ is the peak particle velocity and $p$ is the particle density [21].

We simulated fragmentation for each spherical particle using the Grady's Algorithm [22] shown in eqn (5)

$$
D=\left(\frac{4.472 \times K l_{c}}{\rho V_{p} S_{r}}\right)^{2 / 3}
$$

$D$ is the diameter of the resultant progeny particle, $K l_{c}$ is fracture toughness of the material $\left(\mathrm{Pa} \mathrm{m}^{0.5}\right), V_{p}$ is the propagation velocity $(\mathrm{m} / \mathrm{s})$ of longitudinal elastic waves in the material and $\mathrm{Sr}$ is the induced strain rate. The strain rate is calculated from the rotational velocity of the impeller and the radial distance between the center of the rotation and the impact point.

Table 2: $K$ values of materials used in the study [17].

\begin{tabular}{llll}
\hline Material & Fast-flo lactose & Avicel 101 & Regular lactose \\
\hline$K$ value & 45 & 60 & 75 \\
\hline
\end{tabular}


The peak particle velocity at the contact point of the sample and impeller, for the simplest case, is equal to impact velocity $V_{\mathrm{i}}(\mathrm{m} / \mathrm{s})$. This impact velocity of the impeller is determined by the angular (rotational) velocity of the impeller as well as the distance from the center of rotation where impact occurs and is described by the formula

$$
V_{\mathrm{i}}=\omega d
$$

$\omega$ is the angular velocity of the impeller $(\mathrm{rad} / \mathrm{s})$ and $d$ is the distance from the center along the impeller where collision occurs [12]. Following fragmentation, the resultant particles are considered as spheres, obeying the laws of conservation of total mass and momentum [23].

Powder characteristics relevant to breakage of solid particles such as Young's modulus and fracture toughness were incorporated into the model. The material properties and DEM parameters used in our simulation are shown in Table 3. As comminution is a complicated process and DEM being computationally expensive; we utilized a simplified geometry for our mill. For our preliminary studies, we simulate the flow and fragmentation of 4000 particles of size 5-mm radius under different conditions. The four hammers are simulated by rigidly grouping particles of $2 \mathrm{~mm}$ size, which perform solid body rotations (Fig. 1).

Table 3: DEM parameters for simulation cases.

\begin{tabular}{ll}
\hline DEM parameters & Values \\
\hline Total number of particles & 4000 \\
Radius of the particles & $5 \mathrm{~mm}$ \\
Hopper angle & 72.83 \\
Coefficient of restitution: particle/particle & 0.7 \\
Coefficient of restitution: particle/wall & 0.5 \\
Normal stiffness coefficient: particle/particle & $600 \mathrm{~N} / \mathrm{m}$ \\
Normal stiffness coefficient: particle/wall & $600 \mathrm{~N} / \mathrm{m}$ \\
Friction coefficient: particle/particle & 0.7 \\
Friction coefficient: particle/wall & 0.3 \\
Time step & $2.0 \times 10^{-5}$ \\
Young's modulus & $3.45 \mathrm{GPa}$ \\
Fracture toughness & $0.5-0.8 \mathrm{MPa} \mathrm{m}{ }^{0.5}$ \\
\hline
\end{tabular}

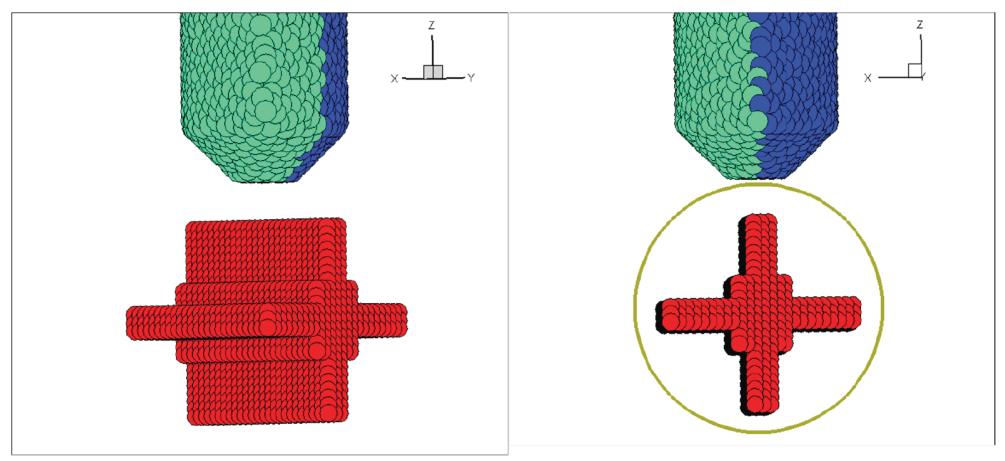

Figure 1: Isometric view (left) and end view (right) of hammer mill in simulations. 


\section{RESULTS AND DISCUSSION}

\subsection{Experimental results}

\subsubsection{Effect of rotational speed of the hammer on milling}

In an ideal milling process, fragment sizes would be mono-dispersed. However, in reality a fragmentation leads to a wide distribution of fragment sizes that can span several orders of magnitude. The speeds of the impeller along with the mechanical properties of material of interest determine the mechanism of size reduction. Harder materials, on virtue of their material property, do not display much attrition at lower impeller speeds. For most brittle particles, lower speeds result in some amount of attrition or surface wear, whereas higher speeds cause size reduction by impact due to catastrophic fragmentation. To investigate the effect of speed, experiments were conducted at 600,870 and $1140 \mathrm{rpm}$ at a given feed rate of $60 \mathrm{~g} / \mathrm{min}$ and $2.9 \mathrm{~mm}$ impeller wall tolerance. Figure 2 depicts the behavior of size reduction upon changing the speed at a given feed rate. As impact initiates rapid propagation of pre-existing flaws, a high speed of the mill produced a smaller particle size and a faster comminution rate. Besides achieving a smaller particle size, a narrower size distribution was obtained at $1140 \mathrm{rpm}$. Thus, the appropriate combination of the feed rate and mill speed can also enable us to produce not only a small size but also control product variability.

\subsubsection{Effect of clearance on milling}

The hammer wall clearance is determined by the distance between the four knives on the wall of mill and on the rotating impeller. A very large distance can result in continuous rolling of particles and hence significantly reduce the rate of size reduction. A very small tolerance, on the other hand, can result in unwarranted fine formation. Figure 3(a) and (b) depicts the behavior of size reduction upon changing the clearance at a given feed rate and speed. This change in clearance significantly influenced the mechanism of size reduction, changing it from impact to a combination of rolling and attrition observed at lower speeds.

As particle wall fragmentation contributes to a greater extent at high impeller speeds, the decrease in comminution rate for $1140 \mathrm{rpm}$ was lower compared with $600 \mathrm{rpm}$.

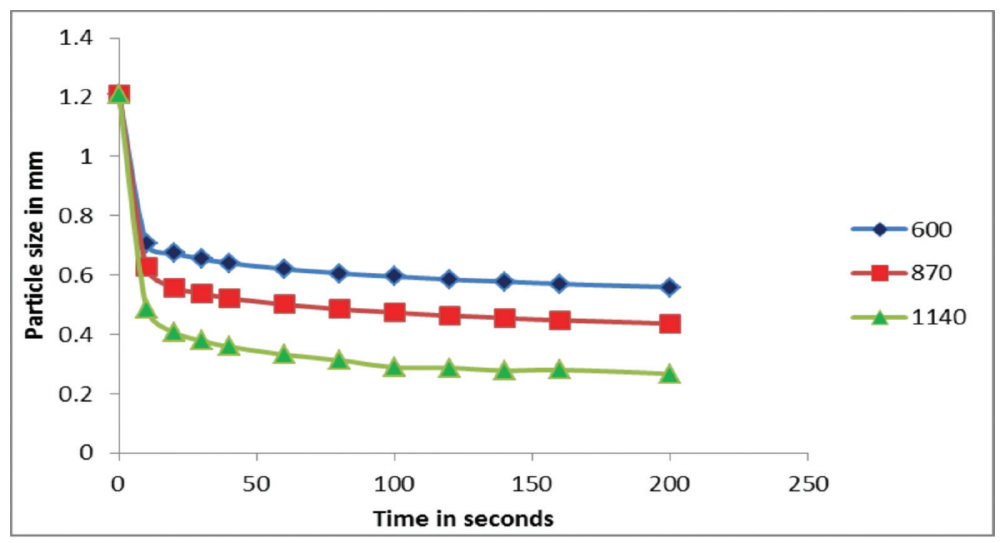

Figure 2: Change of average particle size over time as a function of impeller speed. 


\subsection{Numerical simulations of granular flow and fragmentation}

\subsubsection{Effect of rotational speed of hammer on the milling}

DEM is used to simulate the flow and fragmentation of particles in the hammer mill. Initially, 4000 spherical particles with material properties described in Table 1 are deposited in the hopper. The hopper outlet is chosen to be closed during hopper deposition of particles. After deposition, particles are discharged from hopper into the hammer mill. The hammers are set into motion at prescribed velocity of $600 \mathrm{rpm}$ and/or $1140 \mathrm{rpm}$. Using simulation data, the average kinetic energy of the particles was calculated at different impeller speeds and plotted against time (Fig. 4).

The increase in kinetic energy of particles with an increase in impeller speed is well predicted by the simulation model. The velocity field gradient of the particles present in the mill illustrated in Fig. 5(a) and (b) further corroborates the impact experienced by the particle, which is greatest at the impeller tip. The simulation snapshots in Fig. 6 demonstrate the evolution of the milling process and the formation of greater number of particles at an impeller speed of $1140 \mathrm{rpm}$ compared with $600 \mathrm{rpm}$ implicating catastrophic fragmentation at higher speeds. This is due to the greater impact experienced by the particles while colliding to the hammer rotating at higher speed.

The effect of hammer speed is quantified by counting the number of total particles present within the mill as a function of time for all different speeds. Figure 7 depicts the generation of more particles at higher rotational speeds of the impeller.

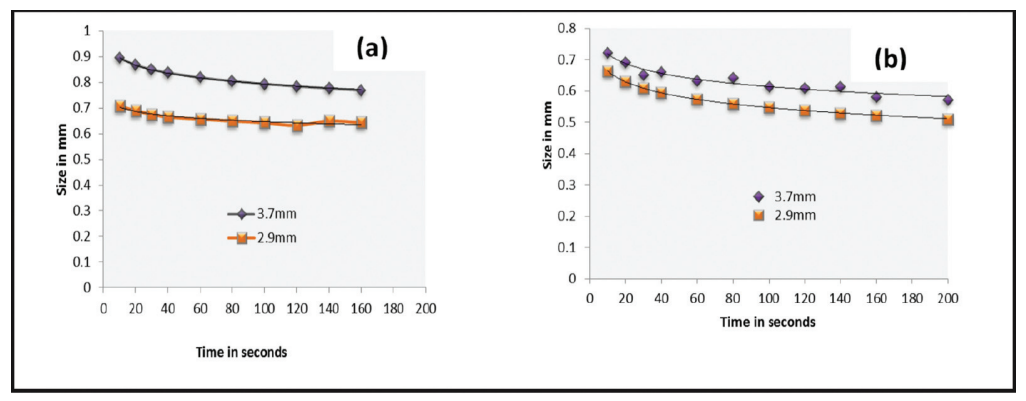

Figure 3: Average particle size with time as a function of wall-hammer tolerance: (a) hammer speed of $600 \mathrm{rpm}$ and (b) hammer speed of $1140 \mathrm{rpm}$.

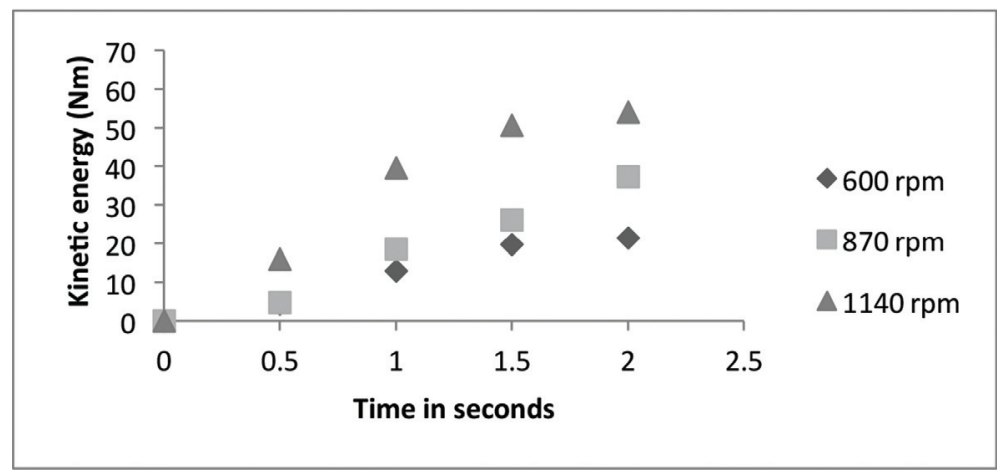

Figure 4: Variation of granular kinetic energy with time as a function of hammer speed. 


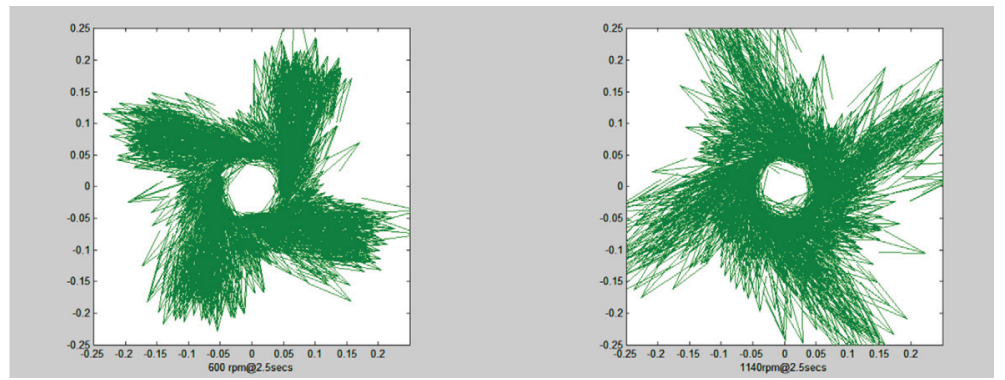

Figure 5: Velocity field profile of particles at (a) $600 \mathrm{rpm}$ and (b) $1140 \mathrm{rpm}$.

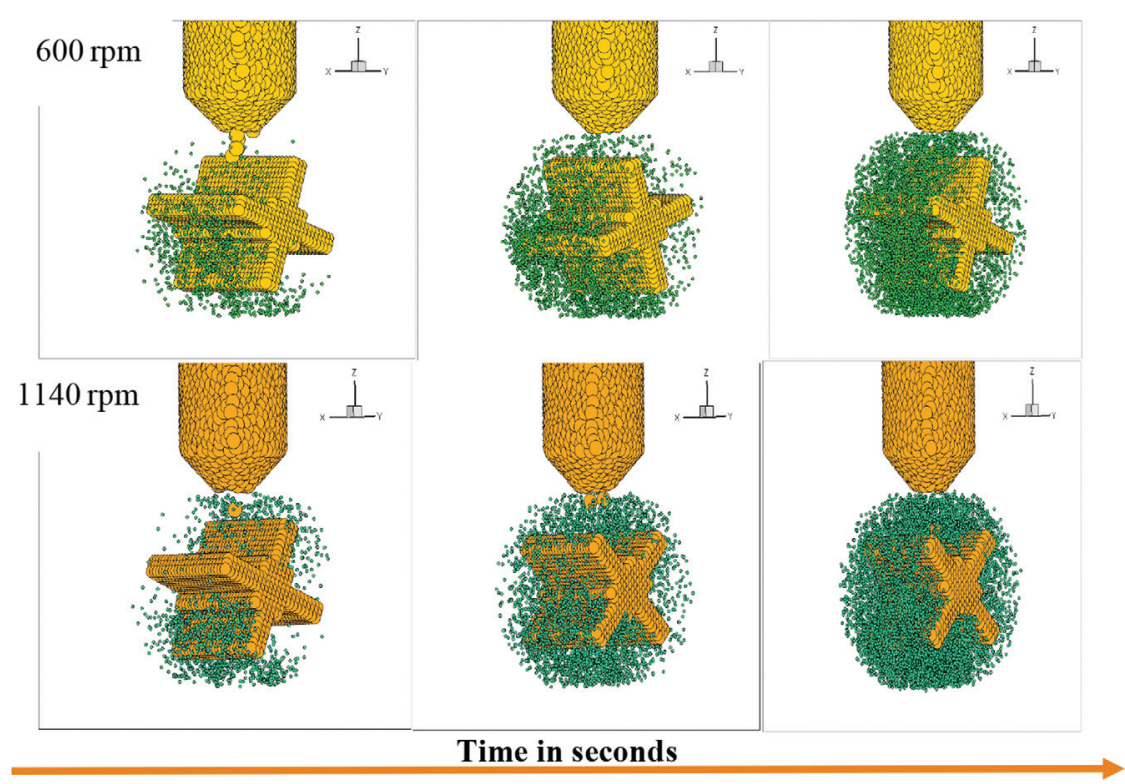

Figure 6: Evolution of particle fragmentation as a function of hammer speed with time.

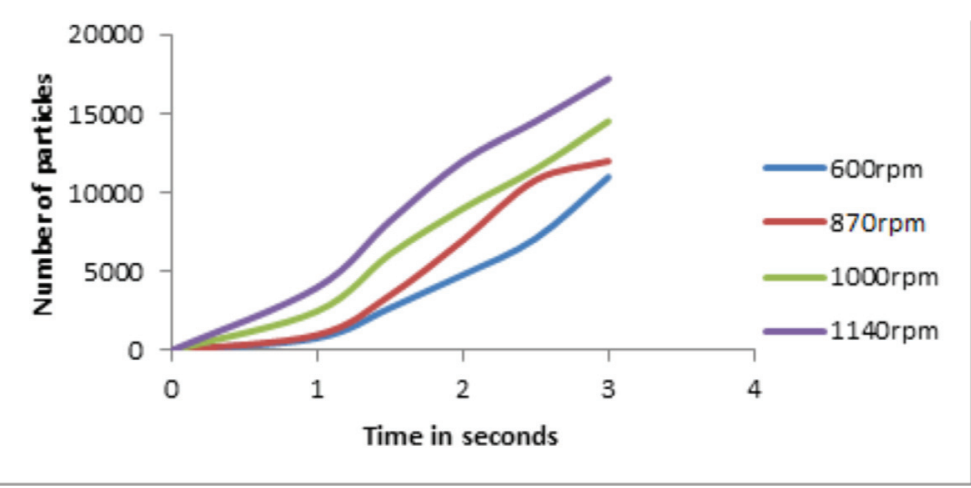

Figure 7: Evolution of particle progeny as a function of hammer speed with time. 


\subsubsection{Effect of hammer-wall clearance on milling}

The effect of impeller wall tolerance was approximated by creating long hammers (5000 particles) and short hammers (2500 particles) thereby providing a hammer-wall tolerance of 3 and $6 \mathrm{~cm}$, respectively. The number of particles present during the milling for both the tolerances is illustrated in Fig. 8(a) and (b) for the hammer speed of 600 and $1140 \mathrm{rpm}$, respectively. As the impeller tolerance increased, the comminution rate slowed down as lesser particles now impact the surrounding hammer. Thus, with the increase in tolerance, particles fragmenting decreased and hence fewer new particles were generated.

\subsubsection{Effect of material properties of granular materials on milling}

The effect of granular cohesion on fragmentation was examined while keeping the operational condition (600 rpm and 2.9-mm clearance) constant. As described in Section 3, materials with different cohesions were modeled using different values of $K$. The milling of the Fast-flo lactose, Avicel 101 and regular lactose was modeled using $K=45,60$ and 75, respectively, following a previously described algorithm [19,20]. Figure 9 (a) and (b) shows the DEM simulation of particle dynamics and fragmentation of Regular Lactose and Fast-flo Lactose, respectively. An intermediate size of particle was observed for Regular lactose,
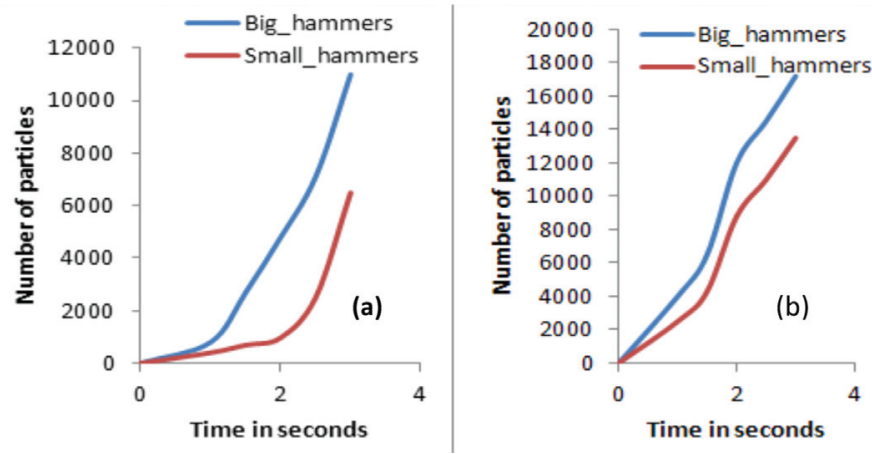

Figure 8: Variation of the number of particles with time as a function of hammer-wall tolerance for hammer speed of (a) $600 \mathrm{rpm}$ and (b) $1140 \mathrm{rpm}$.

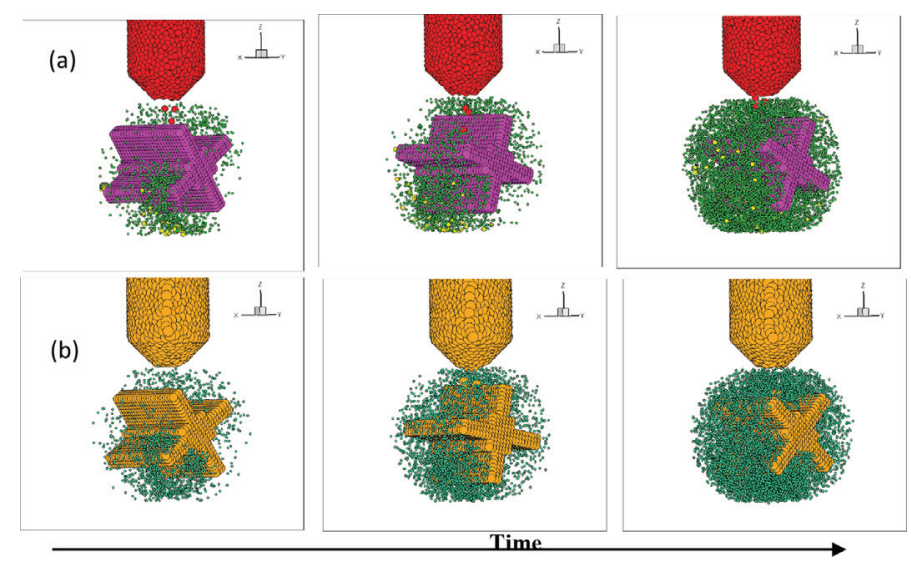

Figure 9: Evolution of particle fragmentation as a function of cohesion over time: (a) regular lactose and (b) Fast-flo lactose. 

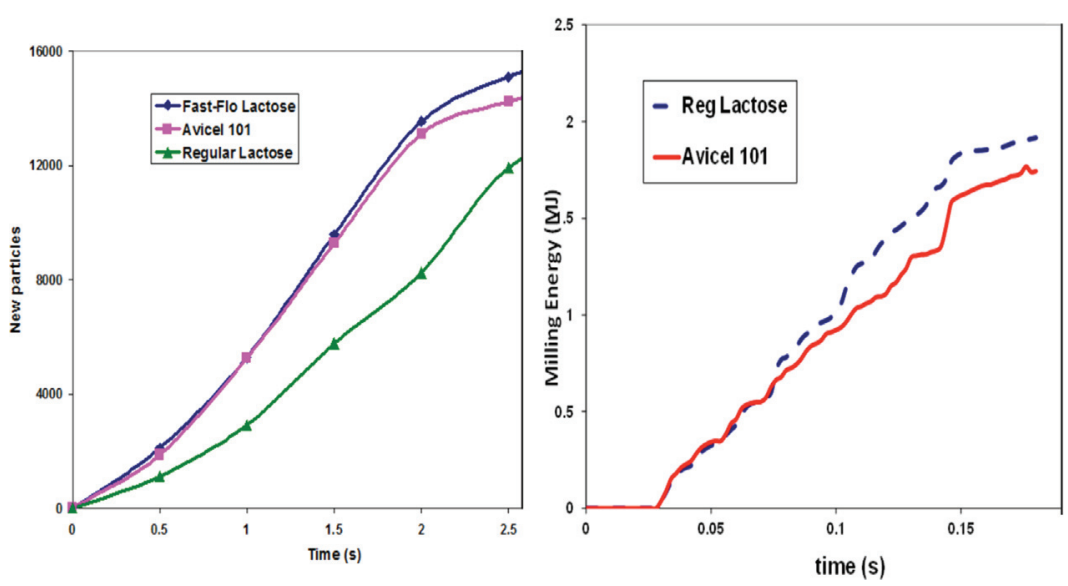

Figure 10: Effect of cohesion by using materials with different $K$ value on (a) fragmentation of particles and (b) energy consumption of mill.

whereas all the Fast-flo lactose particles fragmented into lowermost size of the particle. Material with higher $K$ value has higher cohesion and was found to be more difficult to mill because of their poor flow properties caused by hindered interaction with impeller. This manifests into poor fragmentation thereby reducing the number of new fragments formed by following the impact as for materials of higher cohesion as shown in Fig. 10(a).

The particular observation in the effect of cohesion to the milling behavior was further corroborated by computing the energy of fragmentation at definite time intervals using eqn (6), where $\left(m^{*}\right)$ is the geometric mass of the colliding particles and $\left(v_{j}\right)$ is the relative velocity of the colliding particles from the particle dynamics simulation. Figure 10(b) illustrates that a greater energy of fragmentation (computed from eqn (7)) is required for more cohesive materials.

$$
E_{n t}=\sum_{j=1}^{n} \frac{1}{2} m^{*} v_{j}^{2}
$$

which is Regular Lactose $(K=75)$, as compared with Avicel $101(K=60)$ at impeller speed of $600 \mathrm{rpm}$.

\section{INDUCED STRESS OF IMPACT IN THE HAMMER MILL}

To determine the most predominant location of fragmentation on hammer, the impact stress was determined at the two most likely positions that include the hammer tip and the center of hammer. As illustrated in Fig. 11, the induced stress was found to be higher at the tip of the impeller compared with a position at the middle of the impeller. Thus, most of the breakage may occur at the tip of the impeller as greater stress will produce a greater strain resulting in more breakage.

\section{CONCLUSION}

In this paper, a parametric study was performed in both experiments and simulations to study the effect of different operating conditions on size reduction. As impact initiated rapid propagation of pre-existing flaws, a high speed of the hammer mill produced a smaller particle size and a faster comminution rate. In the experiments, shorter gap between the hammer tip and the wall resulted in higher milling rate. In the simulations, the kinetic energy of particles increased with impeller speed, which contributed to catastrophic fragmentation. Increase in hammer tip speed causes higher frequency of impact of particles per unit time and 


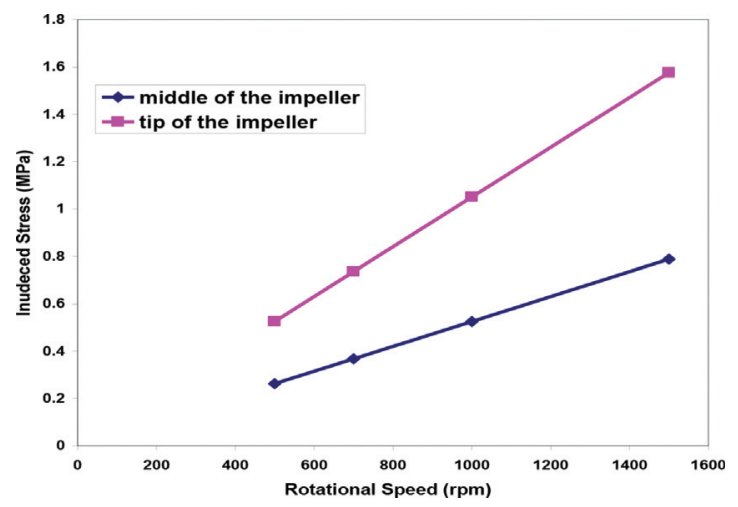

Figure 11: Induced stress computed at two locations on the hammer.

higher specific energy of impact resulting in generation of much finer end product. Simulations also showed higher milling rate for smaller hammer-wall tolerance. In addition, the effect of material properties, viz. cohesion on fragmentation was also studied by simulations. Both the specific kinetic and strain energy of the particles (colliding with hammer) increased as the impact point becomes closer to the hammer tip. Material with lower cohesion showed higher breakage rate than at constant vessel geometry and operating conditions.

\section{ACKNOWLEDGEMENTS}

The authors thank the Centre for Pharmaceutical Processing and Research (CPPR) for funding this research. Dr. Robin Bogner is gratefully acknowledged for her assistance in particle characterization. They also thank the undergraduate students including Jennifer Nguyen, Kirandeep Kaur, Melissa Kuhn, Rhandin DeSantis, Manas Prasad, Nabia Mouli and Emily Butterworth for tirelessly helping in the milling experiments.

\section{REFERENCES}

[1] Jounela, A.J., Pentikainen, P.J. \& Sothmann, A., Effect of particle size on the bioavailability of digoxin. European Journal of Clinical Pharmacology, 8, pp. 365-370, 1975. doi: http://dx.doi.org/10.1007/bf00562664

[2] Clearly, P.W., Predicting charge motion, power draw, segregation, wear and particle breakage in ball mills using discrete element method. Minerals Engineering, 11, pp. 1061-1080, 1998. doi: http://dx.doi.org/10.1016/s0892-6875(98)00093-4

[3] Watanbe, H., Critical rotation speed for ball-milling. Powder Technology, 104, pp. 95-99, 1999. doi: http://dx.doi.org/10.1016/s0032-5910(99)00031-5

[4] Misra, B.K. \& Rajamani, R.K., Simulation of charge motion in ball mills, Part 2: numerical simulations. International Journal of Mineral Processing, 40, pp. 187-197, 1994. doi: http://dx.doi.org/10.1016/0301-7516(94)90043-4

[5] Hlungwani, O., Rikhotso, J., Dong, H. \& Moys, H., Further validation of DEM modeling of milling: effects of linear profile and mill speed. Minerals Engineering, 16, pp. 993-998, 2003. doi: http://dx.doi.org/10.1016/j.mineng.2003.07.003

[6] Austin, L.G. \& Lucker, P.T., A simulation model for air-swept ball mill grinding coal. Powder Technology, 38, pp. 255-266, 1984. doi: http://dx.doi.org/10.1016/00325910(84)85007-X

[7] Kwan, C.C., Mio, H., Chen, Y.C., Ding, Y.L., Saito, F., Papadopoulos, D.G., Benthem, A.C. \& Ghadiri, M., Analysis of the milling rate of pharmaceutical powders using 
distinct element method. Chemical Engineering Science, 60, pp. 1441-1448, 2005. doi: http://dx.doi.org/10.1016/j.ces.2004.10.002

[8] Campbell, G.M., Bunn, P.J., Webb, C. \& Hook, S.C.W., On predicting roller mill performance, Part II: the breakage function. Powder Technology, 115, pp. 243-255, 2001. doi: http://dx.doi.org/10.1016/s0032-5910(00)00349-1

[9] Austin, L., A preliminary simulation model for fine grinding in high speed hammer mills. Powder Technology, 143-144, pp. 240-252, 2004. doi: http://dx.doi.org/10.1016/j.powtec.2004.04.017

[10] Gotsis, C. \& Austin, L.G., Batch grinding kinetics in the presence of a dead space as in a hammer mill. Powder Technology, 41, pp. 91-98, 1985. doi: http://dx.doi. org/10.1016/0032-5910(85)85079-8

[11] Vogel, L. \& Peukert, W., From single particle impact behavior to modeling of impact mills. Chemical Engineering Science, 60, pp. 5164-5176, 2005. doi: http://dx.doi. org/10.1016/j.ces.2005.03.064

[12] Djordjevic, N., Shi, F.N. \& Morrison, R.D., Applying discrete element modeling to vertical and horizontal shaft impact crushers. Minerals Engineering, 16, pp. 983-991, 2003. doi: http://dx.doi.org/10.1016/j.mineng.2003.08.007

[13] Cundall, P.A., A computer model for simulating progressive large-scale movements in blocky rock systems. Proceedings of Symposium International Society of Rock Mechanics, 2, pp. 129, 1971.

[14] Cundall, P.A. \& Strack, O., A discrete numerical model for granular assemblies. Geotechnique, 29, pp. 47-65, 1979. doi: http://dx.doi.org/10.1680/geot.1979.29.1.47

[15] Dippel, S., Batrouni, G. \& Wolf, D., Collision-induced friction in the motion of a single particle on a bumpy inclined line. Physical Review E, 54, pp. 6845, 1995. doi: http:// dx.doi.org/10.1103/physreve.54.6845

[16] Chaudhuri, B., Mehrota, A., Muzzio, F. \& Tomassone, M., Cohesive effects in powder mixing in a tumbling blender. Powder Technology, 165, pp. 105-114, 2006. doi: http:// dx.doi.org/10.1016/j.powtec.2006.04.001

[17] Faqih, A., Chaudhuri, B., Mehrotra, A., Tomassone, M. \& Muzzio, F., Constitutive model to predict flow of cohesive powders in bench scale hoppers. Chemical Engineering Science, 65(10), pp. 3341-3351, 2010. doi: http://dx.doi.org/10.1016/j.ces.2010.02.028

[18] Walton, O.R., Numerical simulation of inclined chute flows of mono disperse, inelastic, frictional spheres. Mechanics of Materials, 16, pp. 239-247, 1993. doi: http://dx.doi. org/10.1016/0167-6636(93)90048-v

[19] Chaudhuri, B., Alexander, A.W.A., Faqih, A, Muzzio, F.J., Davies, C. \& Tomassone, M.S., Avalanching flow of cohesive powders. Powder Technology, 164, pp. 13-21, 2006. doi: http://dx.doi.org/10.1016/j.powtec.2006.01.017

[20] Chaudhuri, B., Muzzio, F. \& Tomassone, M., Modeling of heat transfer in granular flow in rotary vessels. Chemical Engineering Science, 61, pp. 6348-6360, 2006. doi: http:// dx.doi.org/10.1016/j.ces.2006.05.034

[21] Morrison, R.D., Shi, F. \& Whyte, R., Modeling of incremental rock breakage by impact - for use in DEM models. Mineral Engineering, 20, pp. 303-309, 2007. doi: http:// dx.doi.org/10.1016/j.mineng.2006.10.015

[22] Grady, D.E., Fragmentation under impulsive stress loading. Fragmentation by Blasting, ed. W.L. Fourney et al., Society for Experimental Mechanics: Connecticut, USA, pp. 63-72, 1985.

[23] Poschel, T. \& Schwager, T., Computational Granular Dynamics: Model and Algorithms, Springer Verlag: Berlin, Germany, pp. 110, 2005. doi: http://dx.doi.org/10.1007/ s10035-006-0226-5 